\title{
Chemical composition of rape seed from low glucosinolate varieties grown in Poland
}

\section{W. Korol, Teresa Jaśkiewicz, Grażyna Bartuzi, Grażyna Bogusz, Halina Nieściór, C. Grabowski and Elżbieta Mojek}

Central Laboratory of Feed Industry,

Chmielna 2, 20-079 Lublin. Poland

(Received 13 December 1993; accepted 21 February 1994)

\begin{abstract}
Basal nutrients, fatty acids, macro-and microelements, and antinutrients: glucosinolates, tannins and phytates, were determined in 26 samples of sowing seed of 5 varieties of low glucosinolate rape obtained from local branches of Seed Company covering an area of Poland. Amino acids, carotene, and xanthophyll were assayed in pooled samples of each variety. The differences among the samples representing each variety were rather small. Crude protein content was from 19.1 to $22.7 \% \mathrm{DM}$, in Libravo and Bolko varieties, respectively, lysine from 6.48 to $7.30 \mathrm{~g} / 16 \mathrm{~g} \mathrm{~N}$, substantial intervarietal differences in erucic acid content were found; mean value was $0.99 \%$. Aliphatic glucosinolate content ranged from 8.1 in Bolko to $15.7 \mu \mathrm{mol} / \mathrm{g}$ defatted matter in Ceres and was closely correlated with sulphur content. Phytic phosphorus comprised $74.8 \%$ of total $\mathrm{P}$ content.
\end{abstract}

KEY WORDS: low glucosinolate rape seed, amino acids, fatty acids, minerals, antinutrients

\section{INTRODUCTION}

Rape seed production in Poland amounted to 1.043 million tons in 1991, and dropped by about $27 \%$ in 1992 because of unfavourable climatic and economical conditions. Low glucosinolate (LG) rape accounts for about $98 \%$ of total rape production in Poland (Krzymański, 1993). The main varieties grown are: Bolko, Ceres, Liporta, Libravo and Mar. According to Polish Standard (1990) glucosinolate content in LG rape seeds should not exceed 25 micromoles per gram of defatted seed.

Due to an almost total elimination of glucosinolates and erucic acid, the full fat rape seed may be considered as a dietary component for poultry and pigs. However, the level of inclusion of seeds is limited by the residual glucosinolates, tannins, phytate and fibre which decrease its nutritive value.

An assay of chemical composition of LG rape seed grown in North-Eastern Poland by Matyka et al. (1992) did not include the intervarietal comparison. It seemed therefore important to take into account possible changes in seed 
composition associated with development of new varieties of LG rape seed and to repeat the study on the chemical composition of LG rape seed grown in Poland.

\section{MATERIAL AND METHODS}

Twenty-six samples of sowing seed of LG rape obtained from regional stores of Seed Company from all of Poland, were represented 5 varieties: Bolko $(9$ samples), Ceres (7), Liporta (4), Libravo (3), Mar (3). The number of samples per variety was related to the proportion of the variety in total crop of rape seed. Bolko, Ceres, Liporta and Libravo were obtained from 1991 harvest, Mar from 1992.

The seeds were finely ground for analysis, the fat-free samples were prepared for glucosinolate, phytate and tannin determinations.

Basal nutrients were assayed using Weende methods with modifications presented in Poland Standards.

For amino acid (AA) analyses, composite samples were prepared by pooling $5 \mathrm{~g}$ of each sample within the variety. AA concentration was measured using a Beckmann Model 119 CL Liquid Chromatograph with an ion-exchange system after $20 \mathrm{~h}$ hydrolysis in $6 \mathrm{~N} \mathrm{HCl}$ at $110^{\circ} \mathrm{C}$. Methionine and cystine were defermined after oxidation according to Moore et al. (1958) and tryptophan after alkaline hydrolysis according to Miller (1967).

After estrification of FA with a boron trifluoride catalyst in methanol (Matyka, 1976), the fatty acid (FA) composition of lipid fraction was determined by gas chromatography using Hewlett-Packard 8750 Serie II chromatograph.

For macro- and microelement determination, samples were ashed at $550^{\circ} \mathrm{C}$ for about $20 \mathrm{~h}$. The following detection methods were used: a flame atomic absorption spectrometry using the Unicam Model PU 100X spectrometr for calcium $(\mathrm{Ca})$, magnesium $(\mathrm{Mg})$, iron $(\mathrm{Fe})$, zinc $(\mathrm{Zn})$, manganese $(\mathrm{Mn})$, copper $(\mathrm{Cu})$, cobalt $(\mathrm{Co})$, chromium $(\mathrm{Cr})$ and nickel(Ni); the flame emission spectrometry using the above mentioned apparatus for sodium $(\mathrm{Na})$ and potassium $(\mathrm{K})$; colorimetric molybdate method (Fiske and Subbarow, 1925) for phosphorus (P); colorimetric thiocyanate method (Czuba et al., 1970) for molybdenum (Mo) and the Cunningham method with modification of Korol and Boluk (1979) for sulphur (S).

Carotene and xanthophyll were estimated according to La Roche method (Keller, 1988) in composite samples prepared as for AA analysis.

The isothiocyanate level was estimated by gas chromatography (Polish Standard, 1986) and recalculated as glucosinolates. Tannin content in defatted samples was estimated by a spectrophotometric method (Tyczkowska, 1977). 
Phytates were extracted and phytic acid was precipitated with ferric chloride and analysed for P after digestion (Oberleas, 1971).

The data was subjested to analysis of variance.

\section{RESULTS AND DISCUSSION}

With the exception of crude protein and nitrogen-free extractives, the basal nutrient contents did not differ among five varieties of $L G$ rape (Table 1 ). The $C P$ means ranged from 19.1 to $22.7 \%$ DM for Libravo and Bolko varieties,

TABLE 1

Chemical and amino acid composition, $\%$ of dry matter

\begin{tabular}{|c|c|c|c|c|c|c|c|}
\hline \multirow{2}{*}{ Indices } & \multicolumn{5}{|c|}{ Variety } & \multicolumn{2}{|c|}{ Total } \\
\hline & Bolko & Ceres & Liporta & Libravo & Mar & Mean & SD \\
\hline \multicolumn{8}{|l|}{$\begin{array}{l}\text { Chemical } \\
\text { composition }\end{array}$} \\
\hline Dry matter & 93.7 & 94.3 & 94.1 & 93.3 & 93.6 & 93.8 & 0.63 \\
\hline crude protein $(\mathrm{N} \times 6.25)$ & $22.7 \mathrm{a}$ & $20.2 b$ & $21.6 \mathrm{ab}$ & $19.1 b$ & $21.8 a b$ & 21.6 & 1.68 \\
\hline ether extract & 46.7 & 47.2 & 47.3 & 47.5 & 45.9 & 46.9 & 1.25 \\
\hline crude fibre & 6.65 & 7.27 & 7.12 & 6.75 & 7.05 & 6.96 & 0.67 \\
\hline ash & 4.16 & 4.06 & 4.05 & 4.42 & 4.50 & 4.14 & 0.21 \\
\hline $\begin{array}{l}\text { nitrogen free extracti- } \\
\text { ves }\end{array}$ & $19.8 \mathrm{~b}$ & $21.3 \mathrm{a}$ & $19.9 \mathrm{ab}$ & $22.3 \mathrm{a}$ & $20.7 \mathrm{ab}$ & 20.4 & 1.23 \\
\hline \multicolumn{8}{|l|}{ Amino acid } \\
\hline aspartic acid & 1.76 & 1.57 & 1.63 & 1.54 & 1.69 & 1.64 & 0.09 \\
\hline threonine & 1.01 & 0.95 & 0.97 & 0.89 & 0.97 & 0.96 & 0.04 \\
\hline serine & 0.99 & 0.95 & 0.99 & 0.85 & 0.94 & 0.94 & 0.06 \\
\hline glutamic acid & 4.28 & 3.82 & 3.96 & 3.50 & 4.11 & 3.93 & 0.29 \\
\hline glycine & 1.21 & 1.06 & 1.11 & 1.03 & 1.17 & 1.11 & 0.07 \\
\hline alanine & 1.04 & 0.94 & 0.98 & 0.91 & 1.03 & 0.98 & 0.06 \\
\hline cystine & 0.58 & 0.54 & 0.58 & 0.48 & 0.60 & 0.56 & 0.05 \\
\hline valine & 1.19 & 0.96 & 0.97 & 1.07 & 1.17 & 1.07 & 0.11 \\
\hline methionine & 0.44 & 0.43 & 0.45 & 0.42 & 0.48 & 0.44 & 0.01 \\
\hline isoleucine & 0.91 & 0.72 & 0.76 & 0.79 & 0.89 & 0.82 & 0.08 \\
\hline leucine & 1.62 & 1.40 & 1.47 & 1.34 & 1.56 & 1.48 & 0.11 \\
\hline tyrosine & 0.60 & 0.55 & 0.56 & 0.53 & 0.57 & 0.56 & 0.03 \\
\hline phenylalanine & 0.89 & 0.77 & 0.82 & 0.77 & 0.87 & 0.83 & 0.05 \\
\hline lysine & 1.57 & 1.39 & 1.44 & 1.42 & 1.56 & 1.48 & 0.09 \\
\hline histidine & 0.67 & 0.61 & 0.63 & 0.58 & 0.67 & 0.63 & 0.04 \\
\hline arginine & 1.56 & 1.33 & 1.39 & 1.28 & 1.50 & 1.41 & 0.12 \\
\hline tryptophan & 0.24 & 0.24 & 0.24 & 0.20 & 0.24 & 0.23 & 0.02 \\
\hline
\end{tabular}

values in the same line followed by different letters are significantly different $(\mathrm{P}<0.05)$ 
respectively $(P \leqslant 0.05)$. Similar high protein content $(23.3 \% D M)$ in $L G$ rape seed produced in North-Eastern Poland (mainly var. Bolko) was found by Matyka et al. (1992).

The Polish LG rape seed, except var. Bolko, had slightly lower CP than the Canadian rape of Canola type (Nwokolo and Sim, 1989; Sibbald, 1986). The contents of ether extract, crude fibre and ash in Polish rape seed were in agreement with Canadian data, except for lower fibre content than $13.5 \%$ in Canola seed found by Nwokolo and Sim (1989) but this result probably refers to defatted sample.

Differences in AA concentration in seeds (\% DM) were associated with variation in crude protein values (Table 1). The highest levels of AA were found in Bolko and the lowest in Libravo but when expressed as $\mathrm{g} / 16 \mathrm{~g} \mathrm{~N}$ the levels of most essential amino acids were the highest in Libravo. The lysine content was in the range from 1.39 to $1.57 \% \mathrm{DM}$ and from 6.48 to $7.30 \mathrm{~g} / 16 \mathrm{~g} \mathrm{~N}$ (Table 2). Lower values for lysine are given in INRA Tables $(1.21 \%$ and $5.59 \%$; Raw Material Compendium, 1992). Bell and Keith (1991) noted $5.96 \%$ of lysine in rape seed protein.

The fatty acid composition of seeds (Table 3) was rather uniform, but some differences, especially in palmitic, oleic, eicosenoic and erucic acid content were

TABLE 2

Amino acid content, g/16 g N

\begin{tabular}{lccccccc}
\hline \multirow{2}{*}{ Amino acid } & \multicolumn{3}{c}{ Variety } & \multicolumn{2}{c}{ Total } \\
\cline { 2 - 6 } & Bolko & Ceres & Liporta & Libravo & Mar & Mean & SD \\
\hline aspartic acid & 7.56 & 7.56 & 7.30 & 7.88 & 7.56 & 7.57 & 0.42 \\
threonine & 4.32 & 4.57 & 4.38 & 4.57 & 4.35 & 4.44 & 0.19 \\
serine & 4.25 & 4.59 & 4.44 & 4.34 & 4.21 & 4.38 & 0.28 \\
glutamic acid & 18.34 & 18.41 & 17.79 & 17.96 & 18.35 & 18.17 & 1.38 \\
glycine & 5.17 & 5.12 & 4.97 & 5.25 & 5.23 & 5.15 & 0.33 \\
alanine & 4.45 & 4.51 & 4.39 & 4.68 & 4.59 & 4.52 & 0.28 \\
cystine & 2.50 & 2.62 & 2.60 & 2.43 & 2.70 & 2.56 & 0.24 \\
valine & 5.11 & 4.63 & 4.38 & 5.52 & 5.23 & 4.97 & 0.52 \\
methionine & 1.90 & 2.07 & 2.00 & 2.15 & 2.14 & 2.05 & 0.05 \\
isoleucine & 3.91 & 3.48 & 3.39 & 4.05 & 3.98 & 3.76 & 0.38 \\
leucine & 6.92 & 6.75 & 6.64 & 6.89 & 6.99 & 6.84 & 0.52 \\
tyrosine & 2.58 & 2.64 & 2.55 & 2.71 & 2.55 & 2.61 & 0.14 \\
phenylalanine & 3.81 & 3.72 & 3.66 & 3.94 & 3.89 & 3.80 & 0.24 \\
lysine & 6.76 & 6.71 & 6.48 & 7.30 & 7.00 & 6.85 & 0.43 \\
histidine & 2.89 & 2.94 & 2.82 & 2.98 & 2.98 & 2.92 & 0.19 \\
arginine & 6.70 & 6.40 & 6.28 & 6.57 & 6.68 & 6.52 & 0.57 \\
tryptophan & 1.02 & 1.17 & 1.09 & 1.01 & 1.08 & 1.07 & 0.10 \\
\hline
\end{tabular}


TABLE 3

Fatty acids content, $\%$ of total fatty acids

\begin{tabular}{lccccccc}
\hline \multirow{2}{*}{ Fatty acids } & \multicolumn{3}{c}{ Variety } & \multicolumn{2}{c}{ Total } \\
\cline { 2 - 6 } & Bolko & Ceres & Liporta & Libravo & Mar & Mean & SD \\
\hline C 14:0 - myristic & 0.12 & 0.10 & 0.09 & 0.10 & 0.10 & 0.10 & 0.02 \\
C 16:0 - palmitic & $6.79 \mathrm{a}$ & $5.84 \mathrm{~b}$ & $5.13 \mathrm{c}$ & $6.55 \mathrm{ab}$ & $6.18 \mathrm{ab}$ & 6.16 & 0.73 \\
C 16:0 - palmitoleic & 0.65 & 0.60 & 0.49 & 0.54 & 0.57 & 0.60 & 0.09 \\
C 18:0 - stearic & 1.32 & 1.12 & 1.10 & 1.35 & 1.51 & 1.23 & 0.21 \\
C 18:1 - oleic & $54.4 \mathrm{~b}$ & $55.3 \mathrm{~b}$ & $56.8 \mathrm{a}$ & $56.0 \mathrm{ab}$ & $50.9 \mathrm{c}$ & 55.1 & 2.08 \\
C 18:2 - linoleic & 20.6 & 20.8 & 20.2 & 21.0 & 18.9 & 20.5 & 0.74 \\
C 18:3 - linolenic & 11.7 & 10.5 & 11.9 & 10.6 & 10.8 & 11.3 & 0.79 \\
C 20:0 - eicosenoic & 0.92 & 0.83 & 0.86 & 1.04 & 0.93 & 0.89 & 0.07 \\
C 20:1 - eicosenoic & 1.61 & 1.83 & 1.63 & 1.52 & 3.36 & 1.75 & 0.63 \\
C 22:0 - behenic & 0.46 & 0.41 & 0.35 & 0.33 & 0.39 & 0.42 & 0.13 \\
C 22:1 - erucic & 0.54 & 1.79 & 0.76 & 0.37 & 1.50 & 0.99 & 0.36 \\
Others & 0.78 & 0.84 & 0.64 & 0.55 & 0.75 & 0.76 & 0.14 \\
\hline PUFA ${ }^{1}$ & 32.3 & 31.4 & 32.1 & 31.6 & 29.8 & 31.8 & 0.86 \\
\hline
\end{tabular}

${ }^{1}$ polyunsaturated fatty acids, linoleic and linolenic

values in the same line followed by different letters are significantly different $(\mathrm{P}<0.05)$

noted. Sum of polyunsaturated fatty acids (PUFA), linoleic and linolenic, ranged from $29.8 \%$ (Mar) to $32.3 \%$ (Bolko). The $1: 2$ ratio of linolenic to linoleic acid was high as compared with the range from 1:6 to 1:3, recommended for oil used for nutritional purposes (Krzymański, 1993). The average erucic acid content amounted to $0.99 \%$ (similar values were reported by Drozdowski et al. (1990) and Matyka et al. (1992)), however substantial intervarietal differences were found in recent study.

Macro- and microelement contents are shown in Table 4. Ca and $\mathrm{P}$ contents ranged from 3.21 to $3.84 \mathrm{~g} / \mathrm{kg} \mathrm{DM}$ and from 7.25 to $7.70 \mathrm{~g} / \mathrm{kg} \mathrm{DM}$ with the means of 3.38 and $7.35 \mathrm{~g} / \mathrm{kg}$ DM, respectively. S content ranged from 4.43 to 5.41 with the mean $5.00 \mathrm{~g} / \mathrm{kg}$ DM. Other macroelements showed similar range of values. Levels of $\mathrm{Ca}(3.7 \mathrm{~g} / \mathrm{kg} \mathrm{DM}), \mathrm{Mg}(3.2 \mathrm{~g} / \mathrm{kg} \mathrm{DM}), \mathrm{Na}(0.1 \mathrm{~g} / \mathrm{kg} \mathrm{DM})$ and $\mathrm{Mn}(36$ $\mathrm{g} / \mathrm{kg} \mathrm{DM}$ ) reported by Nwokolo and Sim (1989) in full fat Canola seed were similar to the here obtained but those of $\mathrm{Fe}(216 \mathrm{mg} / \mathrm{kg} \mathrm{DM})$ and $\mathrm{Cu}(6.5 \mathrm{mg} / \mathrm{kg}$ DM) were about twice the value found in Polish samples.

The mean content of carotene was 1.6 and of xanthophyll $21.0 \mathrm{mg} / \mathrm{kg} \mathrm{DM}$ (Table 5). The highest levels of these compounds were found in seeds of var. Mar. Similar data concerning the xanthophyll content in Canola seed $(19.2 \mathrm{mg} / \mathrm{kg})$ were reported by Blair and March (1989). 
TABLE 4

Mineral content, $\mathrm{g}$ or $\mathrm{mg}$ per kg dry matter

\begin{tabular}{lclllllll}
\hline \multirow{2}{*}{ Elements } & \multicolumn{3}{c}{ Variety } & \multicolumn{2}{c}{ Total } \\
\cline { 2 - 6 } & Bolko & Ceres & Liporta & Libravo & Mar & Mean & SD \\
\hline $\mathrm{g}$ & & & & & & & \\
$\mathrm{Ca}$ & 3.52 & $3.14 \mathrm{~b}$ & $3.35 \mathrm{ab}$ & $3.21 \mathrm{ab}$ & $3.84 \mathrm{a}$ & 3.38 & 0.30 \\
$\mathrm{P}$ & 7.50 & 7.07 & 7.34 & 7.70 & 7.25 & 7.35 & 0.52 \\
$\mathrm{Mg}$ & $2.82 \mathrm{a}$ & $2.40 \mathrm{~b}$ & $2.65 \mathrm{ab}$ & $2.89 \mathrm{a}$ & $2.78 \mathrm{ab}$ & 2.67 & 0.26 \\
$\mathrm{Na}$ & 0.06 & 0.08 & 0.07 & 0.06 & 0.05 & 0.07 & 0.02 \\
$\mathrm{~K}$ & 8.02 & 8.43 & 7.31 & 8.10 & 8.44 & 8.05 & 0.75 \\
$\mathrm{~S}$ & $4.43 \mathrm{~b}$ & $5.41 \mathrm{a}$ & $5.07 \mathrm{ab}$ & $5.07 \mathrm{ab}$ & $5.00 \mathrm{ab}$ & 5.00 & 0.45 \\
\hline $\mathrm{mg}$ & & & & & & & \\
$\mathrm{Fe}$ & 96.9 & 94.3 & 88.1 & 78.4 & 99.9 & 93.8 & 12.9 \\
$\mathrm{Zn}$ & 43.7 & 52.3 & 57.6 & 41.3 & 46.2 & 48.2 & 17.9 \\
$\mathrm{Mn}$ & 43.6 & 40.5 & 40.7 & 42.7 & 44.4 & 42.1 & 7.8 \\
$\mathrm{Cu}$ & $3.08 \mathrm{a}$ & $2.46 \mathrm{~b}$ & $3.66 \mathrm{ab}$ & $2.68 \mathrm{ab}$ & $3.04 \mathrm{ab}$ & 3.00 & 0.89 \\
$\mathrm{Co}$ & 0.48 & 0.48 & 0.48 & 0.56 & 0.59 & 0.51 & 0.09 \\
$\mathrm{Mo}$ & 0.43 & 0.38 & 0.43 & 0.42 & 0.44 & 0.42 & 0.13 \\
$\mathrm{Ni}$ & 1.04 & 1.04 & 1.03 & 0.84 & 1.27 & 1.04 & 0.31 \\
$\mathrm{Cr}$ & 4.07 & 4.14 & 5.40 & 4.82 & 3.58 & 4.31 & 1.03 \\
\hline
\end{tabular}

values in the same line followed by different letters are significantly different $(\mathrm{P}<0.05)$

TABLE 5

Carotene and xanthophyll content, mg per kg dry matter

\begin{tabular}{lcccccccc}
\hline \multirow{2}{*}{ Indices } & \multicolumn{4}{c}{ Variety } & \multicolumn{2}{c}{ Total } \\
\cline { 2 - 6 } & Bolko & Ceres & Liporta & Libravo & Mar & Mean & SD \\
\hline Carotene & 1.6 & 1.4 & 1.3 & 1.2 & 2.3 & 1.6 & 0.4 \\
Xanthophyll & 22.3 & 19.5 & 19.5 & 16.6 & 27.2 & 21.0 & 4.0 \\
\hline
\end{tabular}

TABLE 6

The content of antinutritional factors

\begin{tabular}{lcccccccc}
\hline \multirow{2}{*}{ Antinutrients } & \multicolumn{4}{c}{ Variety } & \multicolumn{2}{c}{ Total } \\
\cline { 2 - 7 } & Bolko & Ceres & Liporta & Libravo & Mar & Mean & SD \\
\hline Tannin, \% & $1.16 \mathrm{a}$ & $1.00 \mathrm{~b}$ & $1.05 \mathrm{ab}$ & $0.94 \mathrm{~b}$ & $1.02 \mathrm{~b}$ & 1.08 & 0.12 \\
Glucosinolate & & & & & & & \\
$\mu$ mol/g DM defatted & $8.1 \mathrm{~b}$ & $15.7 \mathrm{a}$ & $14.8 \mathrm{ab}$ & $14.6 \mathrm{ab}$ & $14.3 \mathrm{ab}$ & 13.5 & 3.06 \\
Phytatc, \% & $2.08 \mathrm{a}$ & $1.84 \mathrm{~b}$ & $1.89 \mathrm{ab}$ & $1.79 \mathrm{~b}$ & $1.92 \mathrm{ab}$ & 1.95 & 1.6 \\
Phytic phosphorus, \% & $0.57 \mathrm{a}$ & $0.52 \mathrm{~b}$ & $0.54 \mathrm{ab}$ & $0.51 \mathrm{~b}$ & $0.54 \mathrm{ab}$ & 0.55 & 0.4 \\
\hline
\end{tabular}

values in the same line followed by different letters are significantly different $(\mathrm{P}<0.05)$ 
The aliphatic glucosinolate content was low and did not exceed $25 \mu \mathrm{mol} / \mathrm{g}$ of defatted matter in all samples (Table 6). The values ranged from $8.1 \mu \mathrm{mol} / \mathrm{g}$

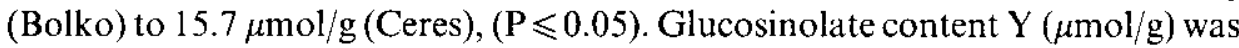
correlated with sulphur content $\mathrm{X}(\mathrm{g} / \mathrm{kg})$ with the respective regression equation:

$$
\mathrm{Y}=8.25 \mathrm{X}-27.7 \pm 0.76, \quad \mathrm{r}=0.955
$$

Mean tannin content was $10.8 \pm 1.2 \mathrm{~g} / \mathrm{kg} \mathrm{DM}$.

Phytate level amounted to $19.5 \mathrm{~g} / \mathrm{kg}$ DM. Phytic phosphorus, poorly utilized by monogastric animals, comprised $74.8 \%$ of total phosphorus content. A ratio of molar content of phytate, expressed as phytic acid, to zinc amounted to 39.6 and was not favourable. At the high inclusion of rape seed in diet, attention should be paid to meeting requirement of animals for zinc. Similar values were obtained in ealier studies (Matyka et al., 1993).

Differences in chemical composition of samples representing each rape variety were rather small, irrespectively of the origin of sample. This indicates the uniformity of the analysed material and shows that the growing conditions in Poland do not affect to a great extent the levels of major nutrients, minerals, and amino acids.

\section{REFERENCES}

Bell J.M., Keith M.O., 1991. A survey of variation in the chemical composition of commercial canola meal produced in Western Candian crushing plants. Can. J. Anim. Sci. 71, 469-490

Blair R., March B.E., 1989. Canola as a source of xanthophyll for poultry diets. Poultry Sci. 68, $1718-1719$

Czuba R., Kamiński W., Strahl A., 1970. Oznaczanie zawartości mikroskładników w materiale roślinnym. Rocz. glebozn. 21, 125-137

Drozdowski B., Hazuka Z., Tynelc M., 1990. Skład jakościowy i ilościowy lipidów nasion rzepaku wybranych odmian podwójnic ulepszonych. Zesz. Probl. IHAR. Rośliny oleiste, Vol. 1, 191-198

Fiske C.H., Subbarow Y., 1925. The colorimetric determination of phosphorus. J. Biol. Chem. 66, 375-379.

Keller H.E., 1988. Analytical methods for vitamins and carotenoids in feed. Hoffman La Roche, Basel p. 75

Korol W., Boluk J., 1979. Oznaczanie siarki w surowcach i mieszankach paszowych metodą absorbcyjnej spektrometrii atomowej. Biul. inf. Przem. Pasz. 18, 31-37

Krzymański J., 1993. Badania genetyczne i hodowlane nad rzepakiem - osiągnięcia i nowe perspektywy. Konferencja naukowa „Rzepak - stan obecny i perspektywy”. Radzików, 3-4 June 1993

Matyka S., 1976. Rutynowa metoda oznaczania zawartości kwasów tłuszczowych. Biul. inf. Przem. Pasz. 15, 38-43

Matyka S., Jaśkicwicz T., Bogusz G., Korol W., 1992. A note on the chemical composition of low glucosinolate rape seed produced in North-Eastern Poland. J. Anim. Feed Sci. 1, 177-182 
Matyka S., Bogusz G., Korol W., 1993. Fityniany w ziarnie zbóż, nasionach roślin strączkowych i rzepaku ,00". Biul. inf. Przem. Pasz. 32, 37-43

Miller E.L., 1967. Determination of the tryptophan content of feedingstuffs with particular reference to cereals. J. Sci. Food Agric. 18, 381-386.

Moore S., Spackman D.H., Stein W.H., 1958. Chromatography of amino acids on sulfonated polystyrene resins. Anal. Chem. 30, 1185-1190

Nwokolo E., Sim J., 1989. Barley and full-fat Canola seed in broiler diets. Poultry Sci. 68, 1374-1380

Oberlcas D., 1971. The determination of phytate and inositol phosphates. Methods Biochem. Anal. 20,87

Polish Standard, 1986. (PN-84) 8054-04. Śruty nasion oleistych: oznaczanie zawartości izotiocyjanianów i 5-winylo-2-oksyzolidynotionu metodą chromatografii gazowej.

Polish Standard, 1990. (PN-90) R-66151. Rośliny przemysłowe oleiste. Ziarno rzepaku i rzepiku podwójnic ulepszonego.

Raw Material Compendium. A compilation of world-wide data sources., 1992. First edition. Novus Europe Sa/nv, Brussels, Belgium

Sibbald I.R., 1986. The TME system of feed evaluation: methodology, feed composition data and bibliography. Animal Research Centre Contribution 85-19 Ottawa, Ontario, Agriculture Canada, pp. 114

Tyczkowska K., 1977. Metoda oznaczania kwasu taninowego w ziarnie sorgo. Biul. inf. Przem. Pasz. $16,40-45$

\section{STRESZCZENIE}

\section{Sklad chemiczny nasion niskoglukozynolanowych odmian rzepaku uprawianego w Polsce}

W 26 próbach nasion 5 odmian niskoglukozynolanowego rzepaku (Bolko, Ceres, Liporta, Libravo i Mar) uzyskanych z terenowych oddziałów „Centrali Nasiennej”, reprezentujących obszar całego kraju, oznaczono zawartość podstawowych skladników pokarmowych, kwasów thuszczowych, makro- i mikroelementów oraz substancji antyżywieniowych: glukozynolanów, tanin j fitynianów. Zawartość aminokwasów oraz karotenu i ksantofilu oznaczono w łączonych próbach nasion każdej z odmian. Stwierdzono niewielkie różnice pomiędzy składem nasion każdej z odmian.

Zawartość białka ogólnego wahała się od 19,1 w odmianic Libravo do $22,7 \%$ suchej masy w odm. Bolko, lizyny - od 6,48 do 7,30 g/16 g N. Stwierdzono istotne różnice między odmianami w zawartości kwasu erukowego; średnia wartość wynosiła $0,99 \%$. Zawartość alifatycznych glukozynolanów wahala się od $8,1 \mathrm{w}$ odm. Bolko do $15,7 \mu \mathrm{mol} / \mathrm{g}$ masy beztłuszczowej w odm. Ceres i była wysoce skorelowana $z$ zawartością siarki. Fosfor fitynowy stanowił $74,8 \%$ fosforu ogólnego. 